\title{
SENTINEL-2 GLOBAL SURFACE REFLECTANCE LEVEL-2A PRODUCT GENERATED WITH SEN2COR
}

\author{
Jérôme Louis ${ }^{(1)}$, Bringfried Pflug ${ }^{(2)}$, Magdalena Main-Knorn ${ }^{(2)}$, \\ Vincent Debaecker ${ }^{(1)}$, Uwe Mueller-Wilm $^{(3)}$, Rosario Quirino Iannone ${ }^{(4)}$, \\ Enrico Giuseppe Cadau ${ }^{(5)}$,Valentina Boccia ${ }^{(6)}$,Ferran Gascon ${ }^{(6)}$
}

(1) Telespazio France, 26 Av. JF Champollion, 31023 Toulouse Cedex 1 (France), email: jerome.louis@telespazio.com, vincent.debaecker@telespazio.com,

(2) German Aerospace Centre, Remote Sensing Technology Institute, email: bringfried.pflug@ dlr.de, magdalena.main-knorn@dlr.de

(3) TPZV-D - Telespazio Vega Deutschland - A Leonardo / Thales Company email: uwe.mueller-wilm@telespazio-vega.de

(4) RHEA SpA, email: rosario.quirino.iannone@esa.int

(5) SERCO Italia SpA, email: enrico.cadau@ serco.com

(6) European Space Agency, ESRIN, Italy email: valentina.boccia@esa.int, ferran.gascon@esa.int

\begin{abstract}
Sen2Cor is a Level-2A processor designed to correct Sentinel-2 Level-1C products from the effects of the atmosphere in order to deliver a Level-2A surface reflectance product. ESA has been using Sen2Cor for systematic Level-2A processing of Sentinel-2 acquisitions over Europe since June 2017. It has since then been successfully integrated into Sentinel-2 ground segment (PDGS) with a global production over the World started in December 2018. In this manuscript, the Level-2A product and algorithm are presented. The performance of this operational Level-2A product is described in terms of cloud screening accuracy and atmospheric correction accuracy. Finally, the on-going parallel developments aimed at improving the product quality at global scale in terms of cloud screening and atmospheric correction are discussed.
\end{abstract}

Index Terms - Atmospheric correction, Cloud screening, Classification, Sentinel-2, Sen2Cor, Validation, Aerosol

\section{INTRODUCTION}

The Sentinel-2 mission is fully operating since June 2017 with a constellation of two polar orbiting satellite units. Both Sentinel-2A and Sentinel-2B are equipped with an optical imaging sensor MSI (Multi-Spectral Instrument) which acquires high spatial resolution optical data products [1]. The Sentinel-2 mission is dedicated to land monitoring, emergency management and security. It serves for monitoring of land-cover change and biophysical variables related to agriculture and forestry, monitors coastal and inland waters and is useful for risk and disaster mapping [2].

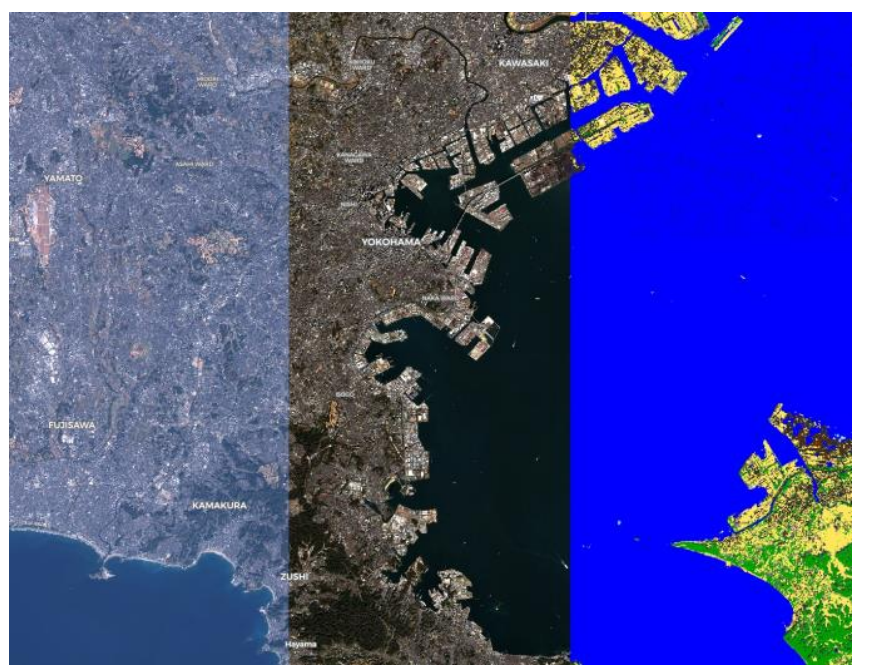

Figure 1: Yokohama region, Japan, Sentinel-2 image acquired on $3^{\text {rd }}$ of January 2019. From left to right: (1) Level-1C TOA reflectance input image, (2) Level-2A surface reflectance image, (3) Level-2A scene classification product. Visualization with EO Browser.

Accurate atmospheric correction of satellite observations is a precondition for the development and delivery of high quality applications and added-value products. Therefore, the atmospheric correction processor Sen2Cor was developed on behalf of ESA. In this perspective Level-2A is considered as a CARD (Copernicus Analysis Ready Data) product. Sen2Cor described in details in [3] is based on Look-Up-Tables computed with libRadtran radiative transfer model [4]. It is designed for mono-temporal processing of Sentinel-2 Level-1C data products providing Level-2A surface (Bottom-of-Atmosphere) reflectance product together with Aerosol Optical Thickness (AOT), Water Vapor (WV) and Scene Classification (SCL) maps. 


\section{CLOUD SCREENING AND SCENE CLASSIFICATION ACCURACY}

Classification accuracy is evaluated by comparison of the Sen2Cor outputs with reference samples. The reference samples are generated by visual inspection and labelling of a validation data set, which was determined by stratified random sampling.

Table 1: Accuracy assessment for SCL product with 11 classes, and for clear pixels, and clouds separation

\begin{tabular}{|l|c|c|c|c|}
\hline \multicolumn{1}{|c|}{ Site } & OA & $\begin{array}{c}\text { OA clear } \\
\text { pixels }\end{array}$ & OA clouds & $\begin{array}{c}\text { Pixel } \\
\text { validated }\end{array}$ \\
\hline Antarctic & 94.7 & 96.8 & 98.8 & 527803 \\
\hline $\begin{array}{l}\text { Barrax } \\
\text { (Spain) -1 }\end{array}$ & 64.6 & 96.9 & 98.7 & 141546 \\
\hline $\begin{array}{l}\text { Barrax } \\
\text { (Spain) -2 }\end{array}$ & 90.5 & 98.7 & 99.5 & 104799 \\
\hline $\begin{array}{l}\text { Berlin } \\
\text { (Germany) }\end{array}$ & 93.4 & 96.5 & no clouds & 51964 \\
\hline $\begin{array}{l}\text { Casleo } \\
\text { (Argentina) }\end{array}$ & 63.8 & 86.1 & 98.1 & 186238 \\
\hline $\begin{array}{l}\text { Dunhuang } \\
\text { (China) }\end{array}$ & 57.3 & 66.2 & no clouds & 105454 \\
\hline $\begin{array}{l}\text { Manila } \\
\text { (Phillipines) }\end{array}$ & 82.1 & 90.0 & 91.6 & 106263 \\
\hline $\begin{array}{l}\text { Rimrock } \\
\text { (USA) }\end{array}$ & 90.2 & 98.2 & 99.2 & 103394 \\
\hline $\begin{array}{l}\text { Yakutsk } \\
\text { (Russia) }\end{array}$ & 69.9 & 93.8 & 92.9 & 177983 \\
\hline $\begin{array}{l}\text { Etna Volcano } \\
\text { (Italy) }\end{array}$ & 95.8 & 97.9 & 99.4 & 132340 \\
\hline $\begin{array}{l}\text { Kilauea Volc. } \\
\text { (Hawaii, U) }\end{array}$ & 60.4 & 75.4 & 74.2 & 118357 \\
\hline $\begin{array}{l}\text { Lagos } \\
\text { (Portugal) }\end{array}$ & 96.8 & 97.3 & no clouds & 69753 \\
\hline $\begin{array}{l}\text { Buenos Aires } \\
\text { (Argentina) }\end{array}$ & 91.8 & 97.3 & no clouds & 31841 \\
\hline $\begin{array}{l}\text { Tallin } \\
\text { (Estonia) }\end{array}$ & 84.3 & 90.4 & 95.6 & 71773 \\
\hline Average & 9.7 & 94.8 & 137822 \\
\hline Stdev & 14.6 & Total: & 1929508 \\
\hline
\end{tabular}

Current analysis of classification accuracy for Sen2Cor 2.5 using auxiliary information from ESA Land Cover Climate Change Initiative (CCI) project [11] is based on 14 Sentinel-2 L2A images over 13 test sites. Validation data set represents different atmospheric conditions (e.g. cloud cover), latitudes (various solar angles and seasons), topography (flat, rough and mountainous terrain), and land cover types (agricultural area, forests, water bodies, arid area, urban area, deserts, permanent ice, and active volcanos).
The accuracy assessment per test site is presented in Table 1. Overall Accuracy (OA) of clear pixels aggregates results for the Sen2Cor classes "vegetation", "nonvegetated" and "water". OA of clouds aggregates results for Sen2Cor classes "cloud medium probability", "cloud high probability" and "thin cirrus". The average OA for 14 classification products reached $81.1 \pm 14.1 \%$. The recognition of clear pixels reached an OA of $91.5 \%$ and a consolidated OA for clouds recognition is $94.8 \%$.

\section{ATMOSPHERIC CORRECTION ACCURACY}

The validation results presented in this chapter were obtained with Sen2cor versions 2.5 (Toolbox version), and may thus slightly differ from the performance of the current Level-2A operational processing baseline PB.02.11. It is intended to update this section with results based on the operational processing baseline PB.02.11.

\subsection{Aerosol Optical Depth accuracy}

Quantitative assessment of aerosol optical depth retrieval accuracy is determined by direct comparison of Sen2Cor output averaged over $9 \mathrm{~km} \times 9 \mathrm{~km}$ region of interest around Sun photometer with reference value from AERONET Sun photometer [5]. The analysis is based on a large dataset of 559 match-ups at 25 AERONET locations distributed over all continents and all climate zones, except Midlatitude South $\left(-30^{\circ} \mathrm{S},-45^{\circ} \mathrm{S}\right)$.

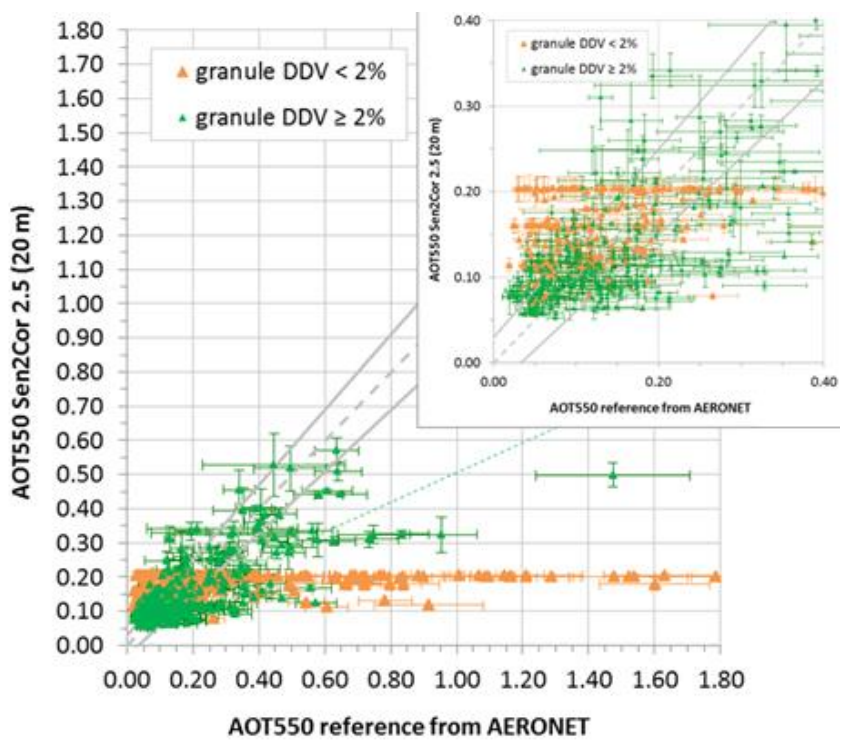

Figure 2: Correlation plot of Sen2Cor AOT550 retrieval at $20 \mathrm{~m}$ resolution over AOT550 reference from AERONET on basis of a data set at 25 AERONET sites. Green triangles are AOT550 retrieved with the DDV-algorithm and orange triangles are AOT550 resulting from the present fallback solution (process with configured start VIS of $40 \mathrm{~km}$ ). The dashed grey line indicates $x=y$ and the solid grey lines show the limits of accuracy requirement $\mid \Delta$ AOT550 $\mid \leq 0.1 \times$ AOT550ref + 0.03. (Insert: zoom on low AOT values). 
Aerosol optical depth (or thickness) retrieval results are very different between the complete data set and the dataset limited to images with at least $2 \%$ of dense dark vegetation (DDV) pixels. The AOT retrieval algorithm implemented in Sen2Cor requires DDV-pixels in the image [3]. If there are not enough DDV-pixels present, then the processing is done with a fixed AOT leading to large AOT errors (Figure 2).

Accuracy \pm precision and uncertainty values are $0.00 \pm$ 0.12 and 0.12 for the DDV subset, which is a very good performance increase since Sen2Cor version 2.4. Correlations are significantly higher for the DDV subset and it contains more retrievals within requirement. Nevertheless, there are only about $50 \%$ of retrievals within requirement.

In order to overcome the limitation of AOT retrieval based on DDV-pixels, a processor evolution is in development to improve the results for arid, snowy, coastal regions where no DDV-pixels are present in the image.

\subsection{Water Vapour accuracy}

Quantitative assessment of water vapour (WV) retrieval accuracy is determined by direct comparison of Sen2Cor output averaged over $9 \mathrm{~km} \times 9 \mathrm{~km}$ region of interest around Sun photometer with reference value from AERONET Sun photometer.

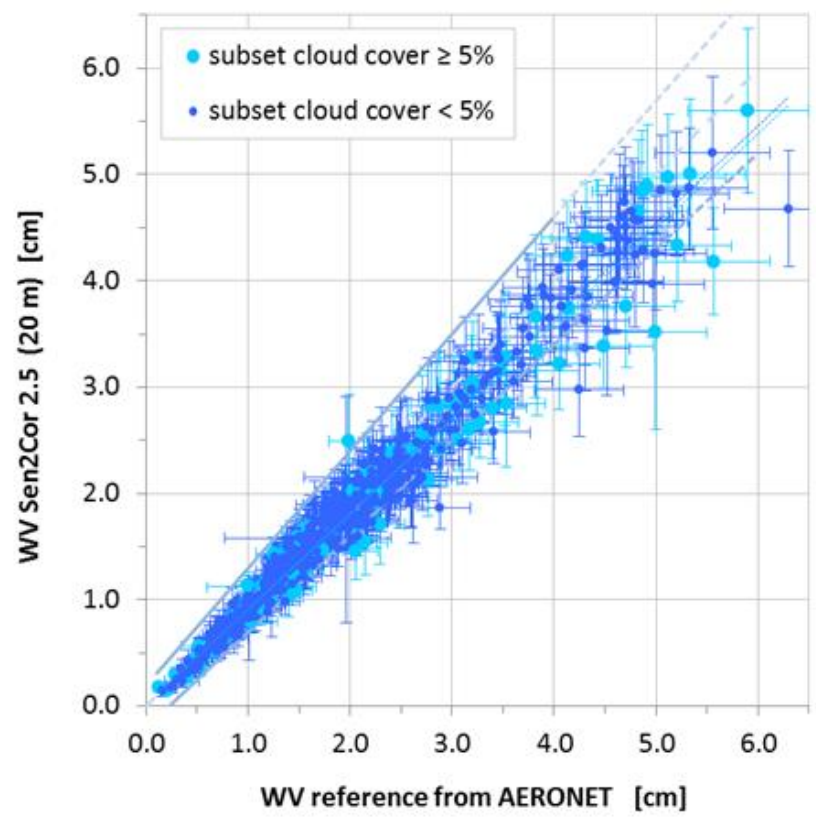

Figure 1: Correlation plot of Sen2Cor WV retrieval at $20 \mathrm{~m}$ resolution over WV reference from AERONET on basis of a data set at 25 AERONET sites. The dashed line indicates $\mathrm{x}=\mathrm{y}$ and the solid lines show the limits of accuracy requirement $|\Delta \mathrm{WV}| \leq 0.1 * \mathrm{WV}$ ref +0.2 .

The analysis is based on a large dataset of 559 match-ups at 25 AERONET locations distributed over all continents and all climate zones except Midlatitude South $\left(-30^{\circ} \mathrm{S},-45^{\circ} \mathrm{S}\right)$.

Water vapour retrieval is very accurate with correlations over 0.98 and with $92 \%$ of retrievals within the requirement.
Validation shows a trend for little underestimation of water vapour by Sen2Cor.

\subsection{Surface reflectance radiometry accuracy}

Quantitative assessment of surface reflectance radiometric performance is provided for Sen2Cor version 2.5 toolbox version. AERONET-corrected surface reflectance data serve as a reference for this analysis. They are computed from the Sentinel-2 Level-1C data (TOA) using the aerosol properties obtained from AERONET in-situ measurements as input to the $6 \mathrm{~S}$ radiation transport processor.

The analysis is based on the dataset defined for the Atmospheric Correction Inter-comparison Exercise (ACIX) [6]. Plots were generated for all Sentinel-2 bands showing the average accuracy, precision and uncertainty values (APU) per surface reflectance bin over all images within the validation data set. Accuracy value is equivalent to the mean bias, precision value is equivalent to the repeatability or variation around the mean bias and uncertainty is quadratic sum of Accuracy and Precision.

Average APU per band is shown in Figure 4.

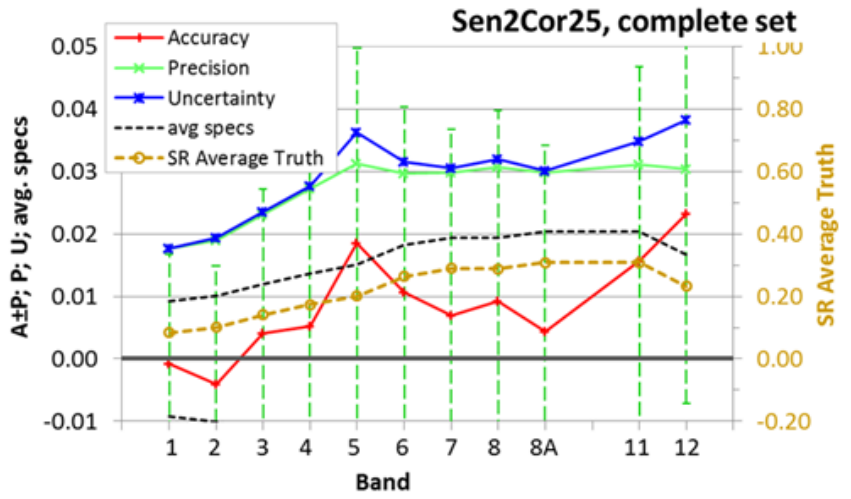

Figure 4: Average accuracy, precision, uncertainty (solid lines, left hand side scale) and surface reflectance reference (dashed line, right hand side scale) per band for Sen2Cor version 2.5 based on the ACIX-dataset.

Accuracy is outside specification for band 5 and band 12 and within specification for all other bands. Both accuracy value and uncertainty show an increasing trend with wavelength (band number). Precision value increases up to band 5 and then stays constant. Thus, best performance is achieved for bands in the visible (VIS) domain with lowest accuracy and precision values and lowest uncertainty.

\section{DISCUSSION}

\subsection{Atmospheric correction improvements}

In case there are not enough DDV-pixels available in the granule, a fallback solution is required. The former fallback solution was to generate an AOT map based on the start visibility set in the configuration file. This fallback solution is one of the limitations of Sen2Cor aerosol retrieval, because it can introduce large errors into the atmospheric 
correction as shown in [7]. In a Sen2Cor prototype version, meteorological AOT estimates from ECMWF Copernicus Atmosphere Monitoring Service (CAMS) [8] are integrated and assimilated into Sen2Cor atmospheric correction to overcome these limitations. The method is described in [9]. Recent results demonstrated noticeable improvements in terms of APU statistics.

\subsection{Cloud screening improvements}

The main limitations of the Cloud Screening and Scene Classification module are: false cloud detection in bright areas like arid regions or cities, false snow detection in high altitude clouds, as well as limited cloud shadow accuracy for complex atmospheric conditions. The topographic shadows classification should be improved in the near future with the usage of a more precise digital elevation model (DEM).

In order to address the issue of false snow detection in clouds that can lead to perturbation of the atmospheric correction itself, the promising research area is related to the particular sensor focal plane geometry. As described in [10], a parallax effect between band B08 and band B8A could be exploited to discriminate medium-high altitude clouds from ground. This information could help to mitigate the false snow detection that occurs when only the spectral shape of the pixel is taken into account.

\section{CONCLUSION}

The performance of the Level-2A product generated by Sentinel-2 ground segment (S2 PDGS) using Sen2Cor is presented in this paper.

In parallel of this operational production assessment, a research and development activity is performed to improve the quality of the L2A products in terms of cloud screening accuracy and atmospheric correction performance.

The evolution based on meteorological AOT estimates is designed to improve the atmospheric correction in case of missing dense dark vegetation pixels in the image. Clearly, the advantage of the integration and assimilation of CAMS aerosol estimates into Sen2Cor atmospheric correction strongly depends on the accuracy of CAMS aerosol data that is expected to improve in the coming years with the launch of several atmospheric compositions EO missions.

Future evolutions for improving the accuracy of the cloud screening are in development.

\section{ACKNOWLEDGMENTS}

We would like to thank the AERONET sun-photometer network and their PIs for their important observations. We would like to thank also the Copernicus Atmosphere Monitoring Service team for supplying aerosol estimates and Eric Vermote for providing the figure 4 based on APU analysis.

\section{REFERENCES}

[1] Drusch, M.; Del Bello, U.; Carlier, S.; et al. (2012). "Sentinel2: ESA's Optical High-Resolution Mission for GMES Operational Services", Remote Sensing of Environment, 120, 25-36

[2] ESA (2012). "Sentinel-2: ESA's Optical High-Resolution Mission for GMES Operational Services", ESA SP-1322/2 March 2012, ESA Communications, ESTEC, PO Box 299, 2200 AG Noordwijk, The Netherlands

[3] Richter, R.; Louis, J.; and Müller-Wilm, U. (2012). „Sentinel-2 MSI-Level 2A Products Algorithm Theoretical Basis Document", S2PAD-ATBD-0001, Issue 2.0, Telespazio VEGA Deutschland $\mathrm{GmbH}$, Darmstadt, Germany

[4] C. Emde, R. Buras-Schnell, A. Kylling, B. Mayer, J. Gasteiger, U. Hamann, J. Kylling, B. Richter, C. Pause, T. Dowling, and L. Bugliaro. The libradtran software package for radiative transfer calculations (version 2.0.1). Geoscientific Model Development, 9(5):1647-1672, 2016

[5] Holben, B.N.; Eck, T.F.; Slutker, I.; Tanré, D.; Buis, J.P.; Setzer, A.; Vermote, E.; Reagan, J.A.; Kaufman, Y.J.; Nakajima, T.; et al. (1998), "AERONET-A federated instrument network and data archive for aerosol characterization", Remote Sens. Env. 66, 1-16, doi:10.1016/S0034-4257(98)00031-5.

[6] G. Doxani et al. (2018), "Atmospheric Correction InterComparison Exercise", Remote Sensing, 10 (352), pp 1-18. DOI: doi:10.3390/rs10020352 ISSN 2072-4292

[7] Gascon F., Bouzinac, C., Thépaut, O., et al. (2017). Copernicus Sentinel-2A Calibration and Products Validation Status, Remote Sens. 2017, 9(6), 584; doi:10.3390/rs9060584

[8] Copernicus Atmosphere Monitoring Service https://www.ecmwf.int/en/about/what-we-

do/copernicus/copernicus-atmosphere-monitoring-service

Accessed on 07/01/2019.

[9] Louis, J.; Pflug, B.; Main-Knorn, M.; Debaecker, V.; MüllerWilm, U.; Gascon, F. (2018) Integration and Assimilation of Meteorological (ECMWF) Aerosol Estimates into Sen2Cor Atmospheric Correction. In: 2018 IEEE International Geoscience and Remote Sensing Symposium (IGARSS), pp. 1864-1897. IGARSS 2018

[10] Frantz, D.; Haß, E.; Uhl, A.; Stoffels J.; Hill, J. (2018) Improvement of the Fmask algorithm for Sentinel-2 images: Separating clouds from bright surfaces based on parallax effects. Remote Sensing of Environment Volume 215, 15 September 2018, Pages 471-481

[11] Land Cover CCI Climate Research Data Package (CRDP) https://maps.elie.ucl.ac.be/CCI/viewer/download.php Accessed on 07/01/2019. 